\title{
Paramorfología y lexicogénesis: ¿un portmanteau imposible?
}

Paramorphology and Lexicogenesis: an impossible portmanteau?

\author{
Raymundo Casas Navarro \\ Universidad Nacional Mayor de San Marcos, Lima, Perú \\ Contacto: jcasasn@unmsm.edu.pe \\ https://orcid.org/0000-0002-6598-2346 \\ Jasmin Ochoa Madrid \\ Universidad Nacional Mayor de San Marcos, Lima, Perú \\ Contacto: jochoam@unmsm.edu.pe \\ https://orcid.org/0000-0001-6353-821X
}

\section{Resumen}

El propósito del presente artículo es dilucidar los entresijos del dinámico proceso de lexicogénesis, con especial incidencia en el término 'feminazi', importado al caudal léxico hispano desde los espacios comunicativos de la lengua inglesa. Se trata de un portmanteau que, al integrar los vocablos 'feminista' y 'nazi', construye una nueva palabra con un fuerte sentido peyorativo, usada frecuentemente en ámbitos discursivos muy variados, sobre todo en los foros polémicos que abundan en las denominadas redes sociales del ciberespacio. Debido a sus insoslayables connotaciones ideológicas, se ha erigido una especie de isoglosa sociolectal: mientras un grupo de hablantes considera que se trata de un portmanteau pernicioso que merece el anatema en el uso idiomático por insertarse en un discurso de odio, otro grupo de hablantes emplea el término sin mayores óbices en la representación lingüística. A partir de la teoría y de la metodología de la fusión conceptual inscrita en el dominio de la moderna semántica cognitiva, exploraremos la naturaleza de la proyección conceptual subyacente en el portmanteau, y trataremos de arribar a una conclusión sobre la pertinencia del sentido emergente del disputado término. En virtud del análisis semántico-cognitivo, podremos explicar la bifurcación en las perspectivas generadas en torno a la pugnaz palabra.

Palabras clave: Lexicogénesis; Portmanteau; Fusión conceptual; Feminazi

\begin{abstract}
We propose to elucidate the internal mechanisms of the dynamic process of lexicogenesis, with special emphasis on the term 'feminazi' (recently coined in Spanish from the English language). It is a portmanteau that, by integrating the words 'feminist' and 'Nazi', a new word with a strong pejorative sense is elaborated. This new word is frequently used in different areas, especially in the polemic forums of social networks. Due to its pervasive ideological connotations, a sort of sociolectal isogloss has
\end{abstract}


been built: while a group of speakers considers it a poisonous portmanteau that deserves banishment from the idiomatic use because it inserts itself into a hate speech, another group of speakers considers it to be basically adequate. From the theory and methodology of the conceptual fusion inscribed in the domain of modern cognitive semantics, we will explore the nature of the underlying projections in the portmanteau, and we will try to obtain a plausible conclusion about the semantic emergence of the word. By virtue of the semantic-cognitive analysis, we will be able to explain the bifurcation in the perspectives of the pugnacious portmanteau.

Keywords: Lexicogenesis; Portmanteau; Conceptual blending; Feminazi

Recibido: 14.12 .19

Aceptado: 12.06.20

\section{Introducción}

El lenguaje sexista expresa un sesgo a favor de un grupo humano en virtud de su condición biológica ligada al sexo y, por ello, tiende a discriminar al otro grupo mediante el empleo de ciertas construcciones o mediante la asunción de actitudes francamente peyorativas (Lei, 2006). Generalmente, el sexismo implica una actitud favorable hacia los varones y una postura contraria a las mujeres; así, la consideración tradicional de la mujer como el "sexo débil" es una típica posición sexista: la mujer es contemplada como un ser defectivo que necesita la protección del hombre y, lo que es más grave, puede ser objeto de ataques escorados hacia la impunidad. El sexismo constituye una ideología que suele anclarse en el lenguaje (Cameron y Kulick, 2003); por ejemplo, la expresión "hombre de la calle" se interpreta neutralmente, pero "mujer de la calle" suele connotar una imagen denigrante sobre la mujer. El presente estudio lleva a cabo una exploración sobre una palabra que se puede considerar sexista (el portmanteau 'feminazi') con las herramientas de la semántica cognitiva.

Aunque, tradicionalmente, se ha visto una cuasisinonimia entre 'género' y 'sexo', la teoría vigente sostiene que existe una diferencia fundamental: el sexo es una categoría biológica y el género se concibe como una construcción social a partir del sexo. En consecuencia, el sexo se puede entender dicotómicamente (masculino/femenino), pero el género es una categoría abigarrada que conlleva una serie de actitudes y conductas, incluso modalidades, que plasman una dinámica y compleja condición (Butler, 1990; Cameron, 2010). 
La cuestión del género en la sociedad ha implicado el desarrollo de varios movimientos, incluso una perspectiva que Coates (2009) denomina "lingüística folklórica”, vale decir, la concepción según la cual las mujeres son más 'apacibles' y los hombres, más 'belicistas' (incluso, hay un best-seller de John Gray: Los hombres son de Marte y las mujeres son de Venus). Pues bien, a partir del libro clásico de Robin Lakoff (1975), la ciencia del lenguaje ha abordado la espinosa cuestión entre lenguaje y género desde perspectivas distintas. El enfoque más reciente pone de relieve el factor dinámico, o construccionista, según el cual el género es una construcción social permanente que puede ampliar su horizonte y que establece una pugna cultural, ideológica y social que se epitomiza en la fórmula: los hablantes ejercen el género (Crawford, 1995).

En el marco teórico desarrollado por Fairclough (1996), el lenguaje se entiende como una práctica social, razón por la cual no es conveniente analizar el lenguaje como un sistema neutral cuyos enunciados tienen la misma fuerza ilocucionaria, independientemente del contexto y de los usuarios. Más bien, se puede sostener que los mismos enunciados son factibles de interpretar de manera muy diferente en virtud de quién los dice y desde qué posición los profiere. Las relaciones asimétricas del poder se observan en la relación, o confrontación, entre los géneros: en castellano, existe la palabra 'uxoricida' (marido que mata a su mujer), pero no existe una palabra para designar a la esposa que mata a su esposo (se propone 'viricida', pero no tiene frecuencia de uso). Se trata de un hecho muy revelador que puede ser una fuente para entender la necesidad conceptual de proponer el constructo de feminicidio como una categoría jurídica para enfrentar una realidad deplorable en el mundo.

Todo lo anterior nos permite enfocar el fenómeno que pretendemos abordar en este trabajo. Debido a que la mujer ha sido invisibilizada por mucho tiempo en la sociedad occidental, la causa feminista ha bregado mucho para ir derribando infranqueables muros, inveterados bloqueos y estólidos prejuicios. Aunque haya todavía un largo camino por recorrer para arribar a una situación edificante con equidad de género, se nota que la causa feminista ha logrado triunfos espectaculares, lo que se puede definir como un ascenso en el empoderamiento femenino (Friedman, 1992). Este empoderamiento implica asumir actitudes y programas con una fuerte decisión y campañas con elevado tono, lo que ha 
causado una reacción de temor en el sector más conservador del poder masculino. Analizar esta dialéctica de conflictos requeriría mucho espacio, por lo que hemos decidido acotar el enfoque hacia una palabra generada en la lengua inglesa, de fácil traslación al idioma español.

Dado que el movimiento feminista ha enarbolado la campaña por el aborto seguro, legal y gratuito, y puesto que esta empresa causa mucho resquemor en los sectores más tradicionales de la sociedad, ha habido un contraataque con la invención del término 'feminazi'. Así, esta palabra ha devenido un arma lingüística en contra del movimiento feminista, porque se basa en una extraña analogía entre este y el Partido Nacionalsocialista Obrero Alemán, conocido como partido nazi, que se entiende como un movimiento irracional que causó estragos temibles a la humanidad. Postulamos que, en el trasfondo del empoderamiento femenino, la palabra 'feminazi' se puede considerar como una isoglosa sociolectal y pragmática que divide a los hablantes en dos grupos marcados: quienes usan el término como una forma de escarnio frente a la causa feminista y aquellos que rechazan el término por considerarlo absurdo y muy ofensivo. Por ende, se configura esta bifurcación sociopragmática:

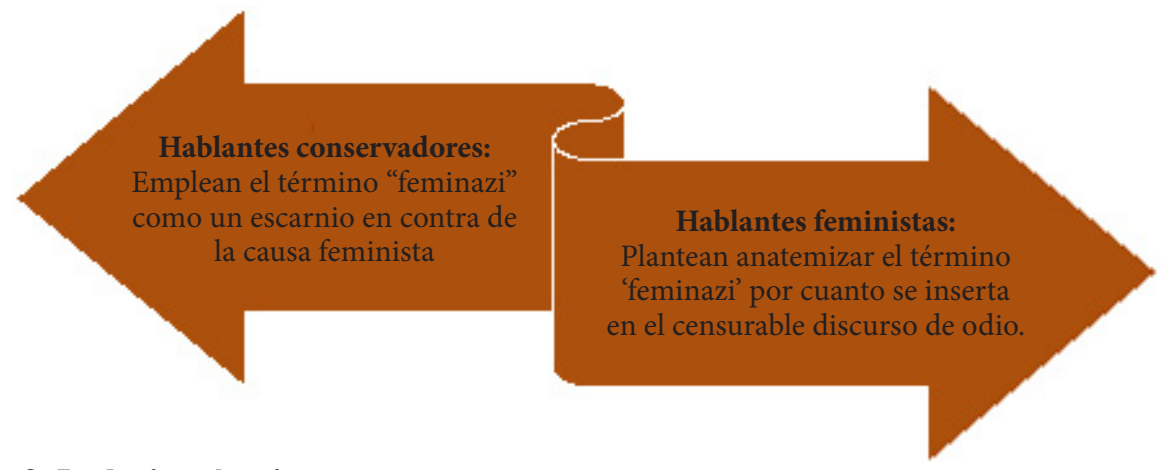

\section{La lexicogénesis}

Desde que la lexicología inició su periplo por el maravilloso mundo del lenguaje, según la plausible expresión de Walter Porzig (1964), se interesó vivamente en los procesos mediante los cuales se enriquece el léxico efectivamente empleado en las diversas comunidades de habla (De Miguel, 2009). La teoría de la lexicogénesis tiene que ver, especialmente, con la explicación acerca de los procesos productivos de formación de palabras, lo que, en última instancia, se 
sustenta en la creatividad del hablante y su respectiva difusión en la comunidad lingüística (Miller, 2014). Así, en virtud del conocimiento tácito del sistema de la lengua, surgen vocablos nuevos por un proceso de fusión (blending), los cuales son denominados palabras portmanteau en la literatura especializada (Katamba, 2005; Yule, 2006). Por ejemplo, a partir de sex + expert, se genera en inglés el siguiente portmanteau: sexpert ('experto en temas sexuales'). Asimismo, hay portmanteau en la palabra ya clásica smog (a partir de smoke + fog).

Migliorini (1975) estableció el constructo parola d'autore o palabras de autor, y postuló una dimensión lexicológica que denominó onomaturgia, esto es, el estudio de las palabras de autor. En nuestra lengua, Unamuno creó la palabra 'noluntad' por analogía con voluntad y sobre la base del latín 'nolo' (no querer). En este contexto, la creación léxica especialmente hecha por un autor o escritor, en tanto proceso onomatúrgico, puede ayudarnos a describir técnicamente el término objeto de nuestra indagación: la palabra 'feminazi'. En efecto, el vocablo fue creado por Tom Hazlett (Limbaugh, 1992) en el idioma inglés y ha sido transferido exitosamente a la lengua castellana (especialmente, su uso es pletórico en redes sociales como Facebook). Al fusionar dos términos ('feminista' y 'nazi'), este portmanteau denigra a un sector radical y pujante del feminismo que recorre el mundo occidental. Así como se observa onomaturgia en filosofía (verbigracia, 'vivencia'), en ciencia (verbigracia, 'escutoide') o en literatura (verbigracia, 'perogrullada'), el proceso se puede hallar en la propia dinámica del lenguaje cotidiano (verbigracia, 'mileurista').

Ahora bien, el feminismo y su crítica (Androne, 2012) han marcado un entorno de ideologías antitéticas en el que, de manera muy controversial, se agudizan visiones contradictorias sobre los roles de género, y esta confrontación se proyecta en el universo simbólico de representaciones en liza. Justamente, en un escenario que propicia la diatriba y el escarnio, la palabra 'feminazi' ha experimentado un sui géneris proceso de ebullición, con usuarios entusiastas e indignados detractores, lo que podría servir para delinear un sistema de actitudes que proyecta una verdadera isoglosa de dimensión sociolectal.

En virtud de un análisis lexicológico profundo, trataremos de esclarecer los mecanismos formales y pragmáticos involucrados en la creación y en la 
difusión de tal 'palabra de autor'. Aunque se puede notar que el uso del término tiene engarces con el fenómeno del ciberodio (online hate speech) (Walker, 1994), nuestra intención es dar cuenta de la referida onomaturgia en función de una explicación basada en los vectores de la cognición, el lenguaje y la cultura.

En los dominios de la sólida teoría lingüística, se ha avanzado mucho en las investigaciones en torno a la interfaz entre semántica y pragmática (Horn y Ward, 2006; Cummings, 2010), especialmente en el análisis de los engarces entre la oración y el enunciado, el cual se concibe como el siguiente par ordenado:

$$
\text { ENUNCIADO }=\{\text { SENTENCIA }+ \text { CONTEXTO DE SITUACIÓN }\}
$$

Asimismo, se puede entablar una interfaz entre morfología y pragmática, sobre todo en el tópico de la formación de palabras o lexicogénesis. Se puede considerar, en efecto, que las estructuras morfológicas suelen estar semánticamente infradeterminadas, razón por la cual su intelección requiere de procesos pragmáticos de índole cognitiva. En una hermosa canción de Charles Aznavour, se habla del "corazón ligero", donde el adjetivo 'ligero' (léger) se proyecta en un sentido encomiástico; pero es fácil barruntar que el adjetivo puede tener otras proyecciones menos halagüeñas. En consecuencia, resulta bienvenida la morfopragmática que, de acuerdo con Kiefer (2004), se define como el estudio de las interrelaciones entre la morfología y la pragmática. Por ejemplo, los desarrollos peyorativos y ameliorativos que experimentan los lexemas dependen crucialmente de un nivel morfopragmático, dado que obedecen a factores contextuales que se despliegan de manera impredecible en la dinámica vida de las palabras.

\section{La paramorfología: el valor del contexto}

En un artículo de posición teórica, Dressler (2000) postula una morfología extragramatical y en ella incorpora tres componentes: la metamorfología (la reflexión deliberada sobre la estructura de las palabras), la premorfología (el modo en que opera la adquisición lingüística; por ejemplo, el fenómeno de la explosión léxica) y la paramorfología. Esta última explora los mecanismos subyacentes que posibilitan el acortamiento (clipping) o la fusión (blend) como operaciones vitales en la lexicogénesis, en tanto y en cuanto proceso dinámico y bullente en 
las lenguas naturales. Según Bauer (2001), un blend o compuesto AB presupone una conexión entre el lexema $\mathrm{A}$ y el lexema $\mathrm{B}$, y este fenómeno se puede dilucidar a partir del significado léxico y de factores eminentemente pragmáticos.

Con el fin de entender un blend cualquiera, se requiere acceder al conocimiento del mundo, al sistema de creencias acerca de la estructura del mundo y a una especie de dimensión axiológica en el caso de los términos cargados emocionalmente, lo que Potts (2007) denomina dimensión expresiva en el uso de los vocablos. En efecto, hay términos que suscitan emociones muy fuertes y este contenido emocional puede acarrear efectos potentes en el procesamiento de las construcciones verbales y en las actitudes hacia los sujetos de la sociedad (Schwab y Schwender, 2011).

Se debe establecer el trasfondo pragmático pertinente, vale decir, un modelo construido a partir del sistema de conocimientos enciclopédicos y del sistema de creencias, valores y actitudes de la sociedad en la que viven los hablantes. La interpretación morfológica requiere activar las presuposiciones pertinentes y cancelar las que no lo son. De esa manera, compuestos sintagmáticos como 'ojo de buey' o 'pata de gallo' implican el doble proceso de activación presuposicional y de cancelación presuposicional (Huang, 2014). Downing (1977) discute un ejemplo muy interesante del inglés: "apple juice seat", es decir, un asiento especial enfrente del cual se coloca una botella de jugo de manzana. Resulta evidente que la configuración léxica de tal expresión no se puede derivar como una mera suma de las palabras que la componen.

En 1872, Lewis Carroll escribió Through the looking glass y acuñó el término portmanteau, muy rentable en la tradición lexicográfica de lengua inglesa. Como lo explica un personaje de la obra, Humpty Dumpty, el portmanteau empaca dos significados en una sola palabra, como acaece con gallop + triumph para generar galumph (galope triunfal). En honor de Lewis Carroll, seudónimo del gran lógico Charles L. Dodgson, en este trabajo usaremos el término portmanteau para hablar de este tipo interesante de fusión léxica.

Ahora bien, para crear un adecuado portmanteau se requiere partir de palabras que sean semántica y fonéticamente compatibles con el fin de efectuar la fusión o el blend, aunque en rigor la creatividad léxica puede sortear ciertos 
óbices conceptuales. En inglés, recientemente, se ha creado frenemy, fusión de friend + enemy, pero ya hay fusiones rentables como townhouse y muy creativas como faction a partir de fact + fiction. En quechua hay portmanteaux como pachamama, yachaywasi o antisuyu. En castellano, es un proceso rentable y con una fuerza de creación expresiva fulminante que puede llegar incluso a ironías atenuadas y sarcasmos zahirientes: 'dedocracia', 'burrocracia', 'dictablanda', 'analfabestia', etc.

\section{El problema de investigación}

Con respecto a un controversial proceso onomatúrgico, nuestro estudio tiene un contexto inicial que se remonta a pocas décadas. El periodista norteamericano Rush Limbaugh, en 1992, escribió un libro The way things ought to be donde refiere que un profesor de Economía, Tom Hazlett, ha acuñado el portmanteau "feminazi", de fácil traslación a la lengua española. Aunque siguiendo a Casado (1999), que quiere ver aquí un caso de acronimia, no nos parece feliz esta descripción, por cuanto la acronimia debiera reservarse para las siglas lexicalizadas (Álvarez de Miranda, 2006).

En rigor, 'feminazi' es un blend o portmanteau (considerado ponzoñoso por las feministas) que resulta de un blending, es decir, de una fusión conceptual, fenómeno rentable en nuestra lengua (Pharies, 1987). En principio, 'feminazi' describiría a las feministas que abogan por una causa radical como el aborto, el mismo que podría considerarse como un trágico holocausto: así como el holocausto en el periodo nazi significó la muerte masiva de seres inocentes, despenalizar el aborto implicaría la extinción de millones de vidas humanas en formación (el nasciturus). El portmanteau opera una fusión conceptual, 'feminista + nazi', y ostenta un uso pletórico en las redes sociales que, luego, ha llegado a otros espacios comunicativos. La definición dada originalmente era la siguiente: "A feminazi is a woman to whom the most important thing in life is seeing to it that as many abortions as possible are performed" (Limbaugh, 1992, p. 195).

¿Es un neologismo el portmanteau 'feminazi'? Guilbert (1975) propuso la siguiente piedra de toque para lo que se puede considerar valor de neologicidad: un neologismo debe superar un periodo de aclimatación de unos diez años, luego del cual se incorpora al léxico de la lengua o simplemente desaparece y es 
enterrado en el cementerio del idioma con honores o sin pena ni gloria. Dado que es un préstamo o importación fácilmente amoldable, no sabemos exactamente cuándo comenzó a emplearse en el habla hispana, pero podemos conjeturar que pasó con éxito la piedra de toque (aunque, como veremos, sufrirá un asedio constante).

En efecto, cuando el término es usado, se colige que se habla de las feministas consideradas más extremistas e intolerantes, y que, en una perspectiva muy estereotipada, concebirían al varón como un potencial acérrimo enemigo de la causa feminista. En tal sentido, el portmanteau 'feminazi' pretende describir una actitud de cerrazón ideológica que se puede considerar irracional, desde un punto de vista neutral u objetivo. Hace poco la Real Academia Española respondió a una consulta sobre el término y pergeñó una mínima definición, lo que fue muy criticado en las redes sociales.

Consideramos que el problema de investigación desplegado por este controversial portmanteau reside en que, en torno a este término, se dan dos antitéticas constelaciones o isoglosas de índole sociolectal:

a) Hablantes que consideran que se trata de un portmanteau imposible porque no hay manera de fusionar los términos 'feminista' y 'nazi'. Son palabras tan incompatibles que la fusión entrañaría una especie de contradictio in adjecto, por lo que su uso caería en el terreno cenagoso de lo absurdo.

b) Hablantes que sostienen que ambos términos son compatibles $\mathrm{y}$, en consecuencia, emplean con naturalidad el portmanteau. Se trata de una fusión posible en la medida en que se puede entender como una analogía o como una hipérbole construida para describir, sarcásticamente, ciertas actitudes feministas consideradas radicales.

Desde un punto de vista neutral o equidistante, podemos constatar simplemente que los usos cargados ideológicamente se dan históricamente en las lenguas. Por ejemplo, en alemán se usaba un portmanteau muy aguerrido, empleado por los conservadores como un insulto lanzado contra los comunistas: Kommunistenschwein. Así pues, podemos determinar una isoglosa entre dos sociolectos. En un sociolecto, el término se emplea de modo peyorativo para 
referirse a un sector de las feministas y no hay ningún óbice conceptual para su uso. En otro sociolecto, se pretende que habría una alotopía en palabras de Klinkenberg (2006), es decir, una grave fisura en el conocimiento enciclopédico que produce una fractura en la isotopía. Así, 'feminazi' deviene en una forma absurda o aberrante, un término que solo expresaría una forma aviesa de pensamiento. Vale decir, en función de los inputs "feminista" y "nazi", no habría modo de llegar al output 'feminazi'.

Teniendo en cuenta lo anterior, arribamos al siguiente problema de indagación: dado que 'feminazi' es un portmanteau que sufre un rotundo rechazo por un sector de hablantes, ¿hay una manera de recusar la fusión, con las herramientas teóricas de la lingüística cognitiva? Siempre en términos de Klinkenberg (2006), ¿podría pensarse en un escollo en el conocimiento enciclopédico que conduciría a una alotopía?

Ahora bien, la batalla ideológica y política se puede ganar, lo que implicaría erigir un sólido anatema contra el portmanteau. En varios países europeos, ya se da un anatema de vocablos que pueden ser hirientes y que incurrirían en el llamado ciberodio. Hay fuertes campañas en contra del online hate speech (Gagliardone, Gal, Alves y Martinez, 2015) y se busca contrarrestar palabras o expresiones cuyo contenido intrínseco atentaría contra el harm principle del liberalismo clásico. Aunque en las democracias occidentales hay una cuasi-irrestricta libertad de expresión, esta libertad tiene que suspenderse si se puede demostrar que se genera un daño basado en la injuria. La perspectiva del liberalismo clásico, empero, siempre ha visualizado dificultades en la aplicación de esta restricción, por cuanto validar un anatema podría permitir la intrusión de la censura arbitraria. Al respecto, una de las opiniones más claras y contundentes es la de Claire Cohen (The Telegraph, 29 de octubre de 2015):

When you choose to call someone a Nazi, there can be no room for doubt or misinterpretation. You are saying that person is the same as the monsters who killed 11 million people. Hitler was Nazi. Goebbels was Nazi. Women who speak up for gender equality are not - and every time they're called feminazi, it diminishes the original term's true meaning.

[Cuando tú dices que alguien es un nazi, no debiera haber espacio para la duda o para la mala interpretación, porque estás aseverando que ese 
sujeto es como esos monstruos que mataron a 11 millones de personas. Hitler fue nazi, Goebbels fue nazi; pero las mujeres que abogan por la equidad de género no lo son, y cada vez que ellas son llamadas feminazis, se está erosionando el verdadero significado del término original.]

Sin embargo, los términos portmanteau, aunque surgen de la fontana de la creatividad lingüística, siempre vienen a cumplir una necesidad designativa, por lo que su uso revela algo acerca de la dinámica del lenguaje y de los usuarios. En Estados Unidos, el anatema que sufrió la palabra 'nigger' no ha significado su completo olvido, y se emplea mucho en las redes sociales. Inclusive, ha sufrido un proceso de bleaching o decoloración semántica: la palabra 'nigga' ha devenido un término que sirve para designar a cualquier hombre, sea negro o caucásico o latino o japonés (Jones y Hall, 2015).

\section{La fusión conceptual: teoría y metodología}

La teoría de la fusión conceptual (Conceptual blending theory) implica una mirada rigurosamente dinámica que puede enlazar, en un todo altamente cohesionado y coherente, la semántica, la cognición y el contexto de las diversas culturas humanas (Fauconnier, 1998; 2000; 2004; 2006). La construcción del significado opera mediante redes semánticas que establecen una serie de engarces, asociaciones e implicancias que posibilitan la emergencia de nuevos conceptos empleados en los diversos entornos comunicativos. En toda asociación, se destacan enlaces contextualmente significativos y se soslayan enlaces que no llegan a puerto: cuando se dice que Popper es el Atila de La inducción, se pone de relieve el carácter destructivo de la crítica popperiana, pero no resulta pertinente vincular al epistemólogo con los hunos desde el punto de vista étnico.

En Fauconnier y Turner (2002) se brindan las bases conceptuales y metodológicas para dilucidar las operaciones cognitivas en los portmanteaux. La fusión conceptual es una miliar operación de la mente humana que nos lleva a un nuevo significado, merced a una nueva inmersión en la estructura de los inputs conceptuales. La fusión conceptual resulta así una útil herramienta cognitiva para la memoria y manipulación de cierta carga semántica, por lo que cumple con el criterio de la economía del lenguaje. Como tal, la fusión conceptual juega un rol esencial en la construcción de los significados de la vida cotidiana. 
Para que se pueda construir una fusión conceptual, debemos operar con tres procesos cruciales: composición, compleción y elaboración (Fauconnier, 1997). En tanto que la composición posibilita que las correspondencias de los inputs originen nuevas relaciones, algunas impredecibles, la compleción permite construir una imagen, experiencialmente inteligible de acuerdo con un marco de asunciones dado por el saber enciclopédico. Finalmente, gracias a la elaboración, se activan las proyecciones que van a converger en el espacio de fusión: el blend (en nuestro caso, el portmanteau).

La dinámica esencial de la fusión conceptual estriba en tres fases:

a) A partir de un espacio genérico, por definición de índole abstracta, se construye una correspondencia parcial entre dos espacios mentales de entrada. En las metáforas prototípicas, la correspondencia parcial se rige por la analogía; en las ironías prototípicas, por la antífrasis según el principio cognitivo de dilución o atenuación.

b) Una vez establecida la correspondencia parcial, se efectúa una proyección selectiva a partir de tales inputs con el fin de hacer emerger un espacio mental nuevo llamado espacio de fusión. Se trata de una proyección selectiva en la medida en que se destacan algunos aspectos de la correspondencia en virtud del principio cognitivo de saliencia o prominencia y se cancelan otros impertinentes o meramente soslayables.

c) De manera dinámica, el espacio de fusión desarrolla una estructura semántica emergente cuyo vehículo es el portmanteau. El significado emergente no se rige por la composicionalidad en la medida en que no es una mera suma de los significados constituyentes.

El método de análisis, en consecuencia, contempla establecer la construcción del sentido, según las líneas directrices del siguiente esquema: 


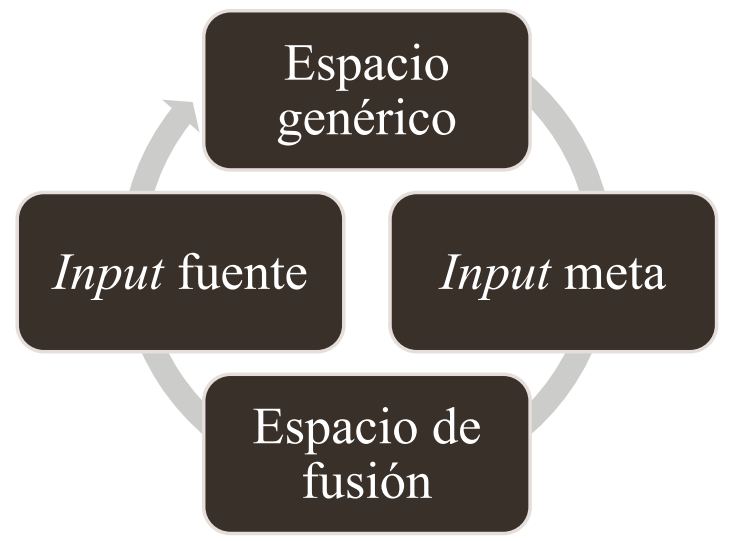

\section{Análisis semántico-conceptual de 'feminazi'}

En Casas Navarro (2019), se sugiere una especie de bifurcación conceptual y actitudinal respecto del portmanteau 'feminazi': hay un uso tolerante frente a un anatema del vocablo. En tanto que un grupo de hablantes opera pertinentemente con la correspondencia y efectúa la proyección, otro grupo de hablantes establece la imposibilidad de llevar a cabo la correspondencia, razón por la cual recusa de plano la fusión conceptual en los meandros de la mente. Esta bifurcación se puede explicar mediante el principio de acceso de la semántica cognitiva. Para que dos elementos entablen la correspondencia en la factoría de la mente, tiene que desarrollarse un acceso cognitivo que se formula así (Fauconnier, 1997):

Si dos elementos $\boldsymbol{a} y \boldsymbol{b}$ se vinculan por un conector $F[b=F(a)]$, entonces el elemento $\boldsymbol{b}$ se puede denominar, describir o señalar mediante su contraparte a.

Para los hablantes que persiguen el anatema, simplemente no hay acceso, dado que no es posible pensar en un conector que vincule los conceptos de 'feminista' y 'nazi'. En cambio, los hablantes que emplean el término, o lo entienden como una hipérbole sarcástica, logran desplegar el principio de acceso y, por ende, establecen la correspondencia como una operación semántica pertinente.

En consecuencia, en quienes emplean el pugnaz portmanteau, se da un acceso regulado por una correspondencia metafórica. En el dominio fuente, se 
sitúa el movimiento nazi y en el dominio meta, el movimiento feminista. Las correspondencias entre ambos dominios tienen que ver con las actitudes radicales de las feministas y con cierta violencia en el escenario de la demanda de derechos. Además, el holocausto perpetrado por los nazis entraña un genocidio de personas inocentes (los judíos), lo que se proyectaría en una posible 'ley' de promoción del aborto, puesto que implicaría una masiva interrupción de la vida de embriones y fetos humanos.

De acuerdo con las líneas teórico-procedimentales de la fusión conceptual, la dinámica del portmanteau entraña realizar la composición, la compleción y la elaboración, tal como se describe a continuación:

\begin{tabular}{|l|l|}
\hline COMPOSICIÓN & $\begin{array}{l}\text { La palabra 'feminista' se une con la palabra 'nazi' en una } \\
\text { operación fonéticamente posible y semánticamente perti- } \\
\text { nente. }\end{array}$ \\
\hline COMPLECIÓN & $\begin{array}{l}\text { Se recurre al saber enciclopédico para ajustar el sentido de } \\
\text { los vocablos anteriores, y se determina que la fusión, en } \\
\text { principio, es posible de acuerdo con ciertos rasgos asocia- } \\
\text { dos al feminismo y al movimiento nazi: intolerancia, acti- } \\
\text { tud violenta, falta de respeto por algunas vidas humanas. }\end{array}$ \\
\hline ELABORACIÓN & $\begin{array}{l}\text { Se genera la fusión conceptual que se fija en el portman- } \\
\text { teau 'feminazi': una feminista radical que exige con vio- } \\
\text { lencia el derecho al aborto. }\end{array}$ \\
\hline
\end{tabular}

En función de este marco conceptual, el caso del portmanteau 'feminazi' implica esta construcción dinámica del sentido:

a) Se postula un espacio mental genérico, el mismo que será la base para las correspondencias entre los espacios mentales de entrada (los inputs). El espacio mental genérico, en este caso, establece la presencia de dos movimientos cualesquiera definidos sobre una base histórica.

b) El espacio mental de entrada o input 1 es la fuente. Se trata de un movimiento definido como nazismo, con sus propiedades pertinentes en la historia de la humanidad. 
c) El espacio mental de entrada o input 2 es la meta. Se trata de un movimiento definido como feminismo, con sus propiedades más conspicuas en la historia social reciente.

d) Entre el input 1 (nazismo) y el input 2 (feminismo) se establecen ciertas correspondencias parciales. Gracias a la saliencia cognitiva, se crea una analogía entre el holocausto de los judíos perpetrado por el nazismo y el aborto propugnado por el feminismo y que implicaría la eliminación de embriones o fetos, es decir, vidas humanas en desarrollo. Por cierto, se trata solamente de correspondencias parciales.

e) A partir de estas correspondencias parciales hay una proyección selectiva para arribar al espacio de fusión. En el espacio de fusión surge el nuevo espacio mental cuyo vehículo es el portmanteau 'feminazi', esto es, prototípicamente una feminista que aboga por el derecho al aborto irrestricto como un modo esencial de la libertad de la mujer.

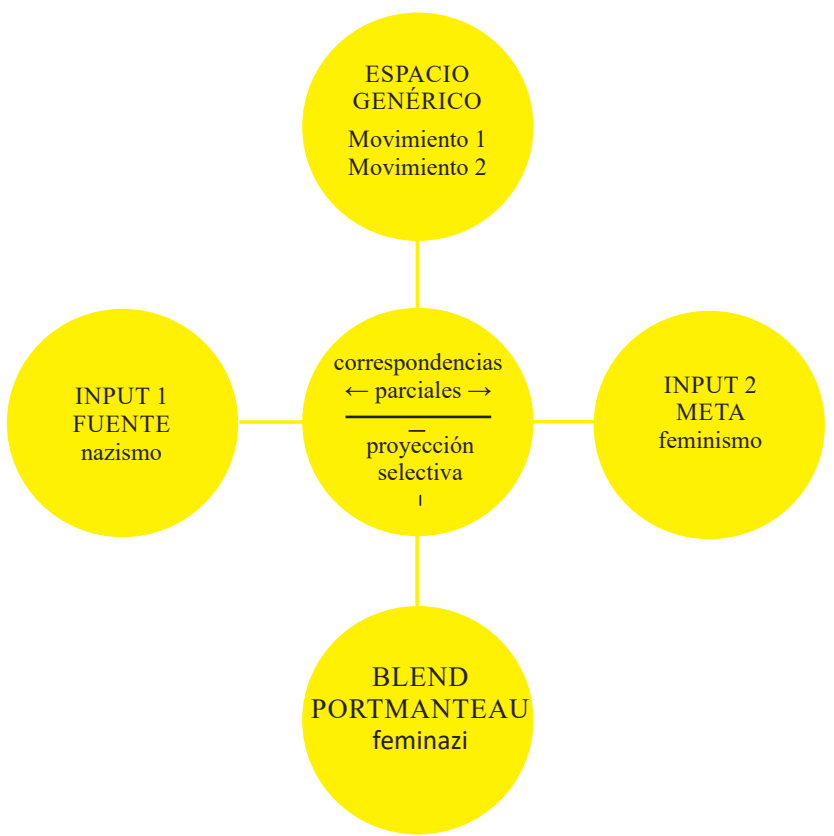




\section{Conclusión}

En el marco de las consideraciones estrictas de la teoría y metodología de la semántica cognitiva, el anatema propugnado por algunas feministas no se puede demostrar inconcusamente. En el portmanteau 'feminazi' no se presupone una identidad entre el 'feminismo' y el 'nazismo', por cuanto toda correspondencia siempre es parcial: solo ciertos rasgos se ponen de relieve. Así, cuando decimos que alguien es un QUIJOTE DE NUESTRO TIEMPO, solo destacamos la lucha idealista por la justicia, y podemos soslayar la enteca complexión de Alonso Quijano. Es decir, en la construcción del sentido de 'feminazi' no habría alotopía en el sentido de una fisura en la cohesión interpretativa del término, por lo que el pretenso descarrilamiento sostenido por la crítica feminista no se puede demostrar con las herramientas de la semántica cognitiva.

No obstante, la fuerte campaña por el anatema del portmanteau 'feminazi' revela que, en la mente de un notable grupo de hablantes, no se despliega el principio de acceso, lo que quiere decir que, desde la cognición feminista, es imposible hacer el engarce entre los conceptos 'feminista' y 'nazi'. Siguiendo la argumentación de Allan y Burridge (2006), los disfemismos, o palabras zahirientes, son como armas que se usan para atacar a otras personas y que funcionan como válvulas de la furia o de la intimidación. La campaña feminista presupone determinar que existen palabras protervas (Battistella, 2005) contra las cuales hay que erigir ciertos óbices legales: la lid trasciende la esfera de la ciencia y debe anidarse en el terreno de la política.

En tal sentido, el anatema propugnado por la causa feminista podría alcanzar el éxito, no en virtud de una teoría de la conceptualización, sino por la vehemencia política del movimiento feminista y el empoderamiento de la causa por la equidad de género. En efecto, si en el mundo occidental se impusiera un derecho al aborto seguro, legal y gratuito, ello tornaría más factible la campaña por el anatema del pugnaz portmanteau.

\section{Referencias bibliográficas}

Allan, K. \& Burridge, K. (2006). Forbidden Words: Taboo and the censoring of language. Cambridge: Cambridge University Press. https://doi. org/10.1017/CBO9780511617881 
Álvarez de Miranda, P. (2006). Acrónimos, acronimia: revisión de un concepto. Biblioteca Virtual Universal. https://biblioteca.org.ar/libros/300007.pdf.

Androne, M. (2012). A terminological analysis of feminist ideology. Procedia Social and Behavioral Sciences, 63, 170-176. https://doi.org/10.1016/j. sbspro.2012.10.026

Battistella, E. (2005). Bad language: Are some words better than others? Nueva York: Oxford University Press.

Bauer, L. (2001). Morphological productivity. Cambridge: Cambridge University Press. https://doi.org/10.1017/CBO9780511486210

Butler, J. (1990). El género en disputa. Barcelona: Paidós.

Cameron, D. (2010). Sex/Gender, Language and the new biologism. Applied Linguistics, 31 (2), 173-192. https://doi.org/10.1093/applin/amp022

Cameron, D. y Kulick, D. (2003). Language and Sexuality. Cambridge: Cambridge University Press. https://doi.org/10.1017/CBO9780511791178

Casado, M. (1999). Otros procesos morfológicos: acortamientos, formación de siglas y acrónimos. En I. Bosque y V. Demonte (Dirs.), Gramática descriptiva de la lengua española (pp. 5075-5096). Madrid: EspasaCalpe.

Casas Navarro, R. (2019). Ironía y cognición. Lima: Editorial Mantaro.

Coates, J. (2009). Mujeres, hombres y lenguaje. Ciudad de México: Fondo de Cultura Económica.

Crawford, M. (1995). Talking difference: On gender and language. Londres: Sage.

Cummings, L. (Ed.). (2010). The pragmatic encyclopaedia. Londres: Routledge. https://doi.org/10.4324/9780203873069

De Miguel, E. (Coord.). (2009). Panorama de la lexicología. Barcelona: Ariel.

Downing, P. (1977). On the creation and use of English compound nouns. Language, 53, 4, 810-842. https://doi.org/10.2307/412913 
Dressler, W. U. (2000). Extragrammatical vs marginal morphology. En U. Doleschal y A. M. Thornton (Eds.), Extragrammatical and Marginal Morphology (pp. 1-10). Múnich: Lincom.

Fairclough, N. (1996). Language and Power. Londres: Longman.

Fauconnier, G.(1997). Mappings in ThoughtandLanguage. Cambridge:Cambridge University Press. https://doi.org/10.1017/CBO9781139174220

Fauconnier, G. (1998). Mental spaces, language modalities, and conceptual integration. En M. Tomasello (Ed.), The New Psychology of Language: Cognitive and Functional Approaches to language structure (pp. 252279). New Jersey: Lawrence Erlbaum.

Fauconnier, G. (2000). Methods and Generalizations. En T. Janssen y G. Redeker (Eds.), Scope and Foundations of Cognitive Linguistics (pp. 95-124). La Haya: Walter De Gruyter. https://doi.org/10.1515/9783110803464.95

Fauconnier, G. (2004). Mental blending and analogy. En D. Gentner, K. Holyoak y B. Kokinov (Eds.), The analogical mind (pp. 255-287). Cambridge: Massachusetts Institute of Technology Press.

Fauconnier, G. (2006). Pragmatics and Cognitive Linguistics. En L. Horn y G. Ward (Eds.), Handbook of Pragmatics (pp. 657-674). Malden: Blackwell. https://doi.org/10.1002/9780470756959.ch29

Fauconnier, G. \& Turner, M. (2002). The way we think: conceptual blending and the mind's hidden complexities. Nueva York: Basic Books.

Friedman, J. (1992). Empowerment: The Politics of Alternative Development. Cambridge: Blackwell.

Gagliardone, I., Gal, D., Alves, T. y Martínez, G. (2015). Countering online hate speech. París: Unesco.

Guilbert, L. (1975). La créativité lexicale. París: Larousse.

Horn, L. y Ward, G. (Eds.). (2006). The Handbook of Pragmatics. Oxford: Blackwell. https://doi.org/10.1002/9780470756959

Huang, Y. (2014). Pragmatics. Nueva York: Oxford University Press. 
Jones, T. \& Hall, Ch. (2015). Semantic bleaching and the emergence of new pronouns in AAVE. LSA, Annual Meeting, 6 (10), 1-5. https://doi. org/10.3765/exabs.v0i0.2994

Katamba, F. (2005). English words. Nueva York: Routledge.

Kiefer, F. (2004). Morphopragmatic phenomena in Hungarian. Acta Linguistica Hungarica, 51, 3-4, 325-349. https://doi.org/10.1556/ ALing.51.2004.3-4.5

Klinkenberg, J. M. (2006). Manual de semiótica general. Bogotá: Universidad de Bogotá.

Lakoff, R. (1975). Language and Woman's Place. Nueva York: Harper \& Row.

Lei, X. (2006). Sexism and language. Journal of Language and Linguistics, 5 (1), 87-94.

Limbaugh, R. (1992). The way things ought to be. Nueva York: Pocket Books.

Migliorini, B. (1975). Parola d'autore. Onomaturgia. Florencia: Sansoni.

Miller, G. (2014). English Lexicogenesis. Oxford. Oxford University Press. https://doi.org/10.1093/acprof:oso/9780199689880.001.0001

Pharies, D. (1987). Blending in spanish word-formation. Romanistisches Jahrbuch, 38, 271-289. https://doi.org/10.1515/9783110244946.271

Porzig, W. (1964). El mundo maravilloso del lenguaje. Madrid: Gredos.

Potts, C. (2007). The expressive dimension. Theoretical Linguistics, 33 (2), 165 197. https://doi.org/10.1515/TL.2007.011

Schwab, F. \& Schwender, C. (2011). The descent of emotions in media: darwininian perspectives. En K. Doveling, C. Scheve y E. A. Konijn (Eds.), The Routledge Handbook of Emotions and Mass Media. Londres y Nueva York: Routledge.

Walker, S. (1994). Hate Speech: the history of American controversy. Nebraska: University of Nebraska Press.

Yule, G. (2006). The Study of Language. Nueva York: Cambridge University Press. 